\title{
Protective effect of Pu-erh tea extracts against ethanol-induced gastric mucosal damage in rats
}

\author{
JINNA YANG ${ }^{1}$, WANGYI ZHOU ${ }^{2}$, YARU GU ${ }^{1}$, JINWEI DAI ${ }^{1}$, XINXIN LI ${ }^{2}$, \\ PING TAI ${ }^{2}$, YANCHUAN LI ${ }^{2}$, XIAOHUI MA ${ }^{2}$ and YUYANG ZHANG ${ }^{1}$ \\ ${ }^{1}$ Department of Pharmacology, School of Life Science and Biopharmaceutics, \\ Shenyang Pharmaceutical University, Shenyang, Liaoning 110016; ${ }^{2}$ Department of Pharmacology, \\ Tasly R\&D Institute, Tasly Pharmaceuticals, Inc., Tianjin 300410, P.R. China
}

Received January 4, 2018; Accepted February 2, 2018

DOI: $10.3892 /$ br.2018.1068

\begin{abstract}
Pu-erh tea has become a focus of research due to its reported biological activities, including anti-oxidation, anti-inflammation and anti-immunosenescence. The present study was performed to evaluate the potential gastroprotective function of $\mathrm{Pu}$-erh tea extracts against ethanol-induced gastric mucosal damage in rats. Sprague Dawley rats were randomly divided into seven groups: A normal control, a model control, a cimetidine $(0.08 \mathrm{~g} / \mathrm{kg})$ group, three $\mathrm{Pu}$-erh tea extracts groups (low, moderate and high-dose; $0.50,1.00$ and $1.50 \mathrm{~g} / \mathrm{kg}$, respectively, and a green tea powder $(1.00 \mathrm{~g} / \mathrm{kg})$ group. The normal and model groups were pre-treated with distilled water while the other groups were respectively administered cimetidine, $\mathrm{Pu}$-erh tea extracts and green tea powder for 14 days. Then, absolute ethanol was orally administered to the rats of all groups excluding the normal controls. The effects of the pretreatments on gastric mucosal injury were evaluated by gross assessment of gastric lesions, examination of histopathology and determination of myeloperoxidase (MPO) activity and asymmetric arginine (ADMA) concentration in gastric mucosal homogenate. Pre-treatment with cimetidine or Pu-erh tea extracts markedly suppressed the formation of ethanol-induced gastric lesions. Furthermore, clear decreases in MPO activity and ADMA concentration in the gastric mucosal homogenate were observed following pretreatment with cimetidine or $\mathrm{Pu}$-erh tea extracts. The anti-gastric ulcer activity of green tea was less than that of Pu-erh tea. Overall, these effects of $\mathrm{Pu}$-erh tea extracts may be due to potential functions in protecting the gastric mucus layer and suppressing inflammation.
\end{abstract}

Correspondence to: Dr Yuyang Zhang, Department of Pharmacology, School of Life Science and Biopharmaceutics, Shenyang Pharmaceutical University, 103 Wenhua Road, Shenyang, Liaoning 110016, P.R. China

E-mail: 13614053862@163.com

Key words: gastric mucosal damage, Pu-erh tea extracts, rats, myeloperoxidase, asymmetric arginine

\section{Introduction}

$\mathrm{Pu}$-erh tea is a type of fermented tea that incorporates microorganism metabolites during the fermentation process (1). It is prepared from processed leaves and buds of the broad-leaf variety of the tea plant [Camellia sinensis var. assamica (L.) O. Kuntze; Theaceae], primarily grown in Yunnan province of China (2). Pu-erh tea is widely consumed worldwide as part of a normal diet. Its chemical components and properties vary due to the time of year that the tealeaves are harvested and the fermentation methods used. The main components of $\mathrm{Pu}$-erh tea extracts include tea polyphenol, tea pigment, tea polysaccharide and alkaloids (3). In previous years, studies on the potential health-beneficial effects of $\mathrm{Pu}$-erh tea have identified a range of biological activities, including anti-oxidation (4), anti-obesity (5), anti-inflammation, anti-immunosenescence (6), anti-hyperlipidemic (7), antitumor (8), antiviral (9) and antibacterial (10) effects. However, its potential action in reducing or limiting gastric mucosal injury and the mechanisms associated with these effects have not been experimentally clarified.

It is established that gastric mucosal injury occurs due to an imbalance between mucosal defensive and aggressive factors (11). Gastric mucosa is frequently exposed to $\mathrm{HCl}$, pepsin, bile acids, ethanol, non-steroidal anti-inflammatory drugs (12), Helicobacter pylori toxins and other noxious substances $(13,14)$. Defensive mechanisms manifest through the activation of various mucosal protection lines, including of mucus and bicarbonate secretion, the mucosal barrier itself, gastric microcirculation (15) and the renin-angiotensin system (16).

The biological functions of $\mathrm{Pu}$-erh tea in the stomach are broad according to previous literature, and include detoxification, promotion of food digestion, regulation of gastrectasia and removal of fats (17). However, there is a lack of data on the gastro-protective activity of $\mathrm{Pu}$-erh tea. In the present study, experiments were designed to investigate the effect and mechanisms of $\mathrm{Pu}$-erh tea extracts on preventing gastric mucosa injury in rats induced with ethanol, namely by histopathological examination and determination of the levels of myeloperoxidase (MPO) and asymmetric arginine (ADMA) in the stomach tissue. MPO activity is regularly used as an indicator for evaluating the progression of intestinal ulcers: it is known to be increased in 
the ulcerated condition and to be reduced through the healing process (18). Meanwhile, it has been demonstrated that ADMA facilitates in gastric mucosal injury: acute administration of high-dose ethanol significantly increased the gastric ulcer index, which was concomitant with an increase of ADMA, while the increased level of ADMA was suppressed by the resveratrol analog BTM-0512 (19). Therefore, these indicators were used in the present study to determine the protective effect of $\mathrm{Pu}$-erh tea extracts against ethanol-induced gastric mucosal damage in rats.

\section{Materials and methods}

Animals. Sprague-Dawley male rats $(\mathrm{n}=126)$ were obtained from the Vital River Laboratories Co., Ltd. (Beijing, China), the weight of which ranged from 180 to $220 \mathrm{~g}$. They were group-housed (5 rats per cage) in standard and pathogen-free environmental conditions $\left(22 \pm 1^{\circ} \mathrm{C}, 60 \pm 5 \%\right.$ humidity, $12-\mathrm{h}$ light/dark cycle) with free access to a standard commercial diet and water ad libitum. Animals were acclimatized to the environment for at least one week. The study protocols were approved by the Tasly Laboratory Animal Welfare and Ethics Committee of Tasly Pharmaceuticals, Inc. (Tianjin, China), and conducted according to the rules of animal experimentation and the guide for the Care and Use of Laboratory Animals of Tasly Pharmaceuticals, Inc. The rats were randomly divided into seven groups ( $\mathrm{n}=18$ per group).

Chemicals and drugs. An aqueous extract of $\mathrm{Pu}$-erh tea was provided by Tasly Pharmaceuticals, Inc., which was dissolved in distilled water and prepared as described previously (1).

The ingredients in fermented $\mathrm{Pu}$-erh tea include caffeine, polyphenols, $\gamma$-aminobutyric acid, theanine, statin, polysaccharides $(3,20-22)$, theaflavins, thearubigins and theabrownins (23-26).

Cimetidine, used as a reference drug in this study, was obtained from GlaxoSmithKline (Shanghai, China) and dissolved in distilled water. A standardized powder of green tea was purchased from Damin Foodstuff (Zhangzhou) Co., Ltd., (Zhangzhou, China). Hematoxylin and eosin were provided by Muto Pure Chemicals Co., Ltd. (Tokyo, Japan). Absolute ethanol, formalin, paraffin and dimethylbenzene were supplied by Rionlon (Tianjin) Industry Co., Ltd. (Tianjin, China). ELISA kits for rat MPO (catalogue no. CK-E30635) and rat ADMA (catalogue no. CK-E30769) were provided by Shanghai Bogoo Biotechnology Co., Ltd. (Shanghai, China). Other reagents used in the study were of analytical grade or higher without further purification.

Ethanol-induced gastric lesion and pharmacological intervention. The rats in normal and model groups were intragastrically (i.g.) administered $10 \mathrm{ml} / \mathrm{kg}$ distilled water, while those in other groups were administered $0.08 \mathrm{~g} / \mathrm{kg}$ cimetidine (i.g.), Pu-erh tea extracts at $0.50,1.00$ and $1.50 \mathrm{~g} / \mathrm{kg}$, and $1.00 \mathrm{~g} / \mathrm{kg}$ green tea powder, respectively. The animals were administered with the test drugs or distilled water between 8:00-9:00 am once a day for 14 consecutive days. Acute gastric lesions were created by intragastric application of absolute ethanol according to a common method (27). The rats, excluding those in the normal control group, were orally administrated absolute ethanol $(5 \mathrm{ml} / \mathrm{kg})$ on day 15 after being fasted for $24 \mathrm{~h}$ but with free
Table I. Gross scoring system for gastric mucosal lesions

\begin{tabular}{lcccc}
\hline & \multicolumn{4}{c}{ Points } \\
\cline { 2 - 5 } Gastric mucosal lesion & 1 & 2 & 3 & 4 \\
\hline Spot erosion (no.) & 1 & - & - & - \\
Erosion length (mm) & $1-5$ & $6-10$ & $10-15$ & $>15$ \\
Erosion width (mm) & $1-2$ & $>2$ & - & - \\
\hline
\end{tabular}

access to water. At $60 \mathrm{~min}$ after ethanol administration, the rats were euthanized by cervical dislocation following an overdose of diethyl ether anesthesia (28-30) and the stomachs were immediately excised. Each stomach was opened along the greater curvature as described by Balan et al (31) and rinsed in cold saline solution. Half of the stomachs in each group were fixed in $10 \%$ buffered formalin at room temperature for $15 \mathrm{~min}$ for anatomical examination, while the others were immediately preserved in liquid nitrogen $\left(-196^{\circ} \mathrm{C}\right)$ for determination of MPO and ADMA.

Gross assessment of gastric lesions. After $15 \mathrm{~min}$, the stomachs in $10 \%$ buffered formalin were removed. Photographs of the gastric mucosa were taken, and the mucosal lesions were scored by a laboratory animal technician blinded to the experimental protocol. The length and width of each injured area of the gastric mucosa were measured with a vernier caliper. Gastric mucosal ulcer index (UI) was determined according to Table I, following the guidelines of the Technical Standards for Testing and Assessment of Health Food (32), and calculated according to the following formula: $\mathrm{UI}=$ spot erosion point + erosion length points + (erosion width points $\mathrm{x} 2)$. The inhibitory rate $(\mathrm{I} \%)$ was calculated by the formula: $\mathrm{I}(\%)=$ [(UIcontrol - UItreated)/UIcontrol] x 100\% (33).

Histopathological examination. The formalin-fixed stomach tissues were embedded in paraffin wax and gradient dehydrated in increasing concentrations of ethanol $(70-100 \% \mathrm{v} / \mathrm{v})$. The specimens were sectioned (4- $\mu \mathrm{m}$ thick) and stained with hematoxylin $(\sim 1.5 \mathrm{~min})$ and eosin $(30 \mathrm{sec})$ at room temperature for histopathological examination. An epithelial damage scoring system (34) was applied to rate histopathological changes, including congestion, edema, hemorrhage, degeneration and necrosis in the gastric mucosa, by light microscopy (x400), as described in Table II. The total score of pathological changes was calculated by the formula: Total score $=$ hyperemia points + (bleeding points $\mathrm{x} 2)+($ degeneration and necrosis points x3) (34).

Determination of MPO and ADMA. The segments of stomach tissue with ulcers were processed into $20 \%$ tissue homogenate in cold saline with a UP400S ultrasonic processor (Ningbo Xinzhi Bio-tech Co., Ltd., Ningbo, China). The rat MPO and ADMA ELISA kits were then used to determine MPO activity and ADMA concentration in the homogenate, according to the manufacturer's protocols. Optical absorbance (O.D.) at $450 \mathrm{~nm}$ was recorded with a Tecan Infinite 200 Microplate Reader (Tecan Group, Ltd., Mannedorf, Switzerland). MPO 
Table II. Scoring system for histopathological changes in gastric mucosal epithelia.

\begin{tabular}{lccccc}
\hline & \multicolumn{5}{c}{ Points } \\
\cline { 2 - 5 } Pathology, area affected & 1 & 2 & 3 & 4 & 5 \\
\hline Hyperemia & $<1 / 5$ & $1 / 5-2 / 5$ & $2 / 5-3 / 5$ & $3 / 5-4 / 5$ & All over the epithelia \\
Bleeding & $<1 / 5$ & $1 / 5-2 / 5$ & $2 / 5-3 / 5$ & $3 / 5-4 / 5$ & All over the epithelia \\
Degeneration and necrosis & $<1 / 5$ & $1 / 5-2 / 5$ & $2 / 5-3 / 5$ & $3 / 5-4 / 5$ & All over the epithelia \\
\hline
\end{tabular}
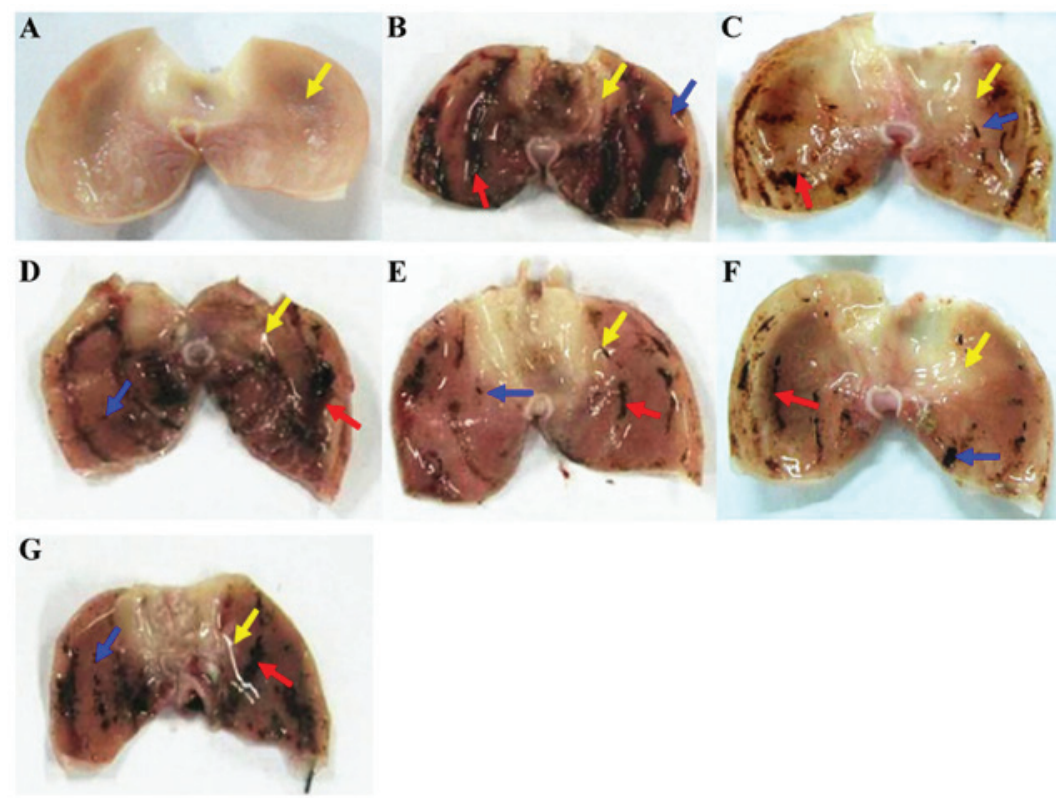

Figure 1. Gross appearance of the gastric mucosa of rats. Representative images are shown (n=9). (A) Treatment with distilled water (normal control). No disturbance in the gastric mucosa was observed. (B) Alcohol-induced gastric lesion pretreated with distilled water (model control). Severe injuries were observed in the gastric mucosa. Absolute ethanol induced extensive visible hemorrhagic necrosis in the gastric mucosa of the rats. (C) Alcohol-induced gastric lesion pretreated with $0.08 \mathrm{~g} / \mathrm{kg}$ cimetidine. Injury in the gastric mucosa was reduced compared with that in the model control. (D) Alcohol-induced gastric lesion pretreated with $1.00 \mathrm{~g} / \mathrm{kg}$ green tea powder. (E-G) Alcohol-induced gastric lesions pre-treated with Pu-erh tea extracts at doses of $0.50,1.00$ and $1.50 \mathrm{~g} / \mathrm{kg}$, respectively. Gastric mucosal injury was reduced in a dose-dependent manner. Blue and red arrows indicate areas of spot erosion and area erosion, respectively; yellow arrows indicate mucosal folds.

activity or ADMA concentration in the samples was then determined by comparing the O.D. value of the samples to the standard curve.

Statistical analysis. The results from each group were expressed as the mean \pm standard error of mean. The data were analyzed by one-way analysis of variance with Fisher's least significant difference post hoc analysis. Statistical analysis was performed with SPSS 16.0 (SPSS, Inc., Chicago, IL, USA), and $\mathrm{P}<0.05$ was considered to indicate statistical significance.

\section{Results}

Protective effect of the extracts in gastric lesions based on gross evaluation. Cimetidine and moderate-to-high-dose $\mathrm{Pu}$-erh tea extracts $(1.00$ and $1.50 \mathrm{~g} / \mathrm{kg})$ administered prior to alcohol-induced gastric injury significantly decreased the ulcer index in rat gastric mucosa, compared with the model control $(\mathrm{P}<0.01$; Figs. 1 and 2). From initial observations, it was noted that the middle-to-high doses of Pu-erh tea extracts markedly suppressed the formation of damage; a notable

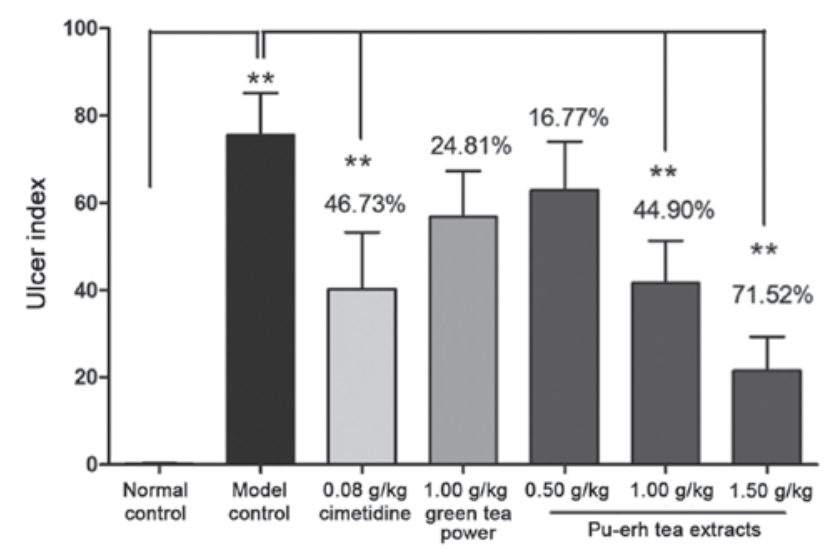

Figure 2. Test drugs reduce ulcer index dose-dependently in rats with alcohol-induced gastric ulcer. Data were expressed as means \pm standard deviation $(n=9)$. ${ }^{*} \mathrm{P}<0.05$ and ${ }^{* *} \mathrm{P}<0.01$ as indicated. The inhibitory rate of each test drug is presented above the bars.

phenomenon was that mucosal folds in the rat stomach became flatter following treatment with the extracts $(0.50-1.50 \mathrm{~g} / \mathrm{kg}$; 

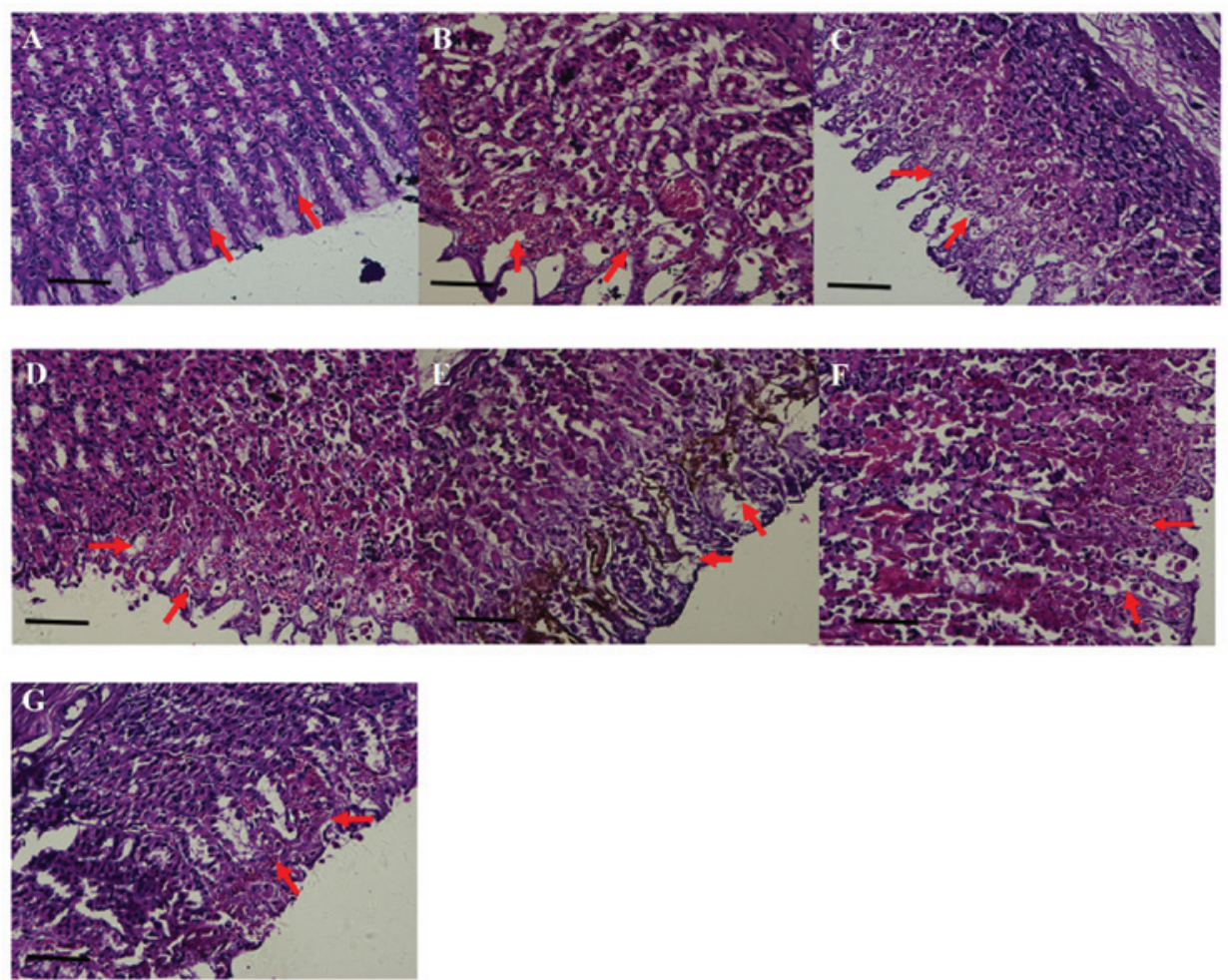

Figure 3. Histopathological evaluation of the gastric mucosa in rats. Representative images are shown (n=9). (A) Treatment with distilled water (normal control). No disturbance in the gastric mucosa or damage in the mucous superficial layer was observed. (B) Alcohol-induced gastric lesion pretreated with distilled water (model control). Severe pathological changes including hyperemia, bleeding and epithelial cell degeneration and necrosis were observed in the gastric mucosal layer. (C) Alcohol-induced gastric lesion pretreated with $0.08 \mathrm{~g} / \mathrm{kg}$ cimetidine. The pathological changes in the gastric mucosa layer were reduced compared with those in the model control. (D) Alcohol-induced gastric lesion pretreated with $1.00 \mathrm{~g} / \mathrm{kg}$ green tea powder. (E-G) Alcohol-induced gastric lesions pretreated with Pu-erh tea extracts at doses of $0.50,1.00$ and $1.50 \mathrm{~g} / \mathrm{kg}$, respectively. The pathological changes in the gastric mucosa layer were inhibited in a dose-dependent manner. The rat stomach sections were stained with hematoxylin and eosin. Images are shown at magnification, $\mathrm{x} 400$. Scale bars, $60 \mu \mathrm{m}$. Red arrows indicate areas of mucosal damage.

Fig. 1). Additionally, the severity of ethanol-induced gastric mucosal damage appeared to be dose-dependently reduced by the pretreatment with Pu-erh tea extracts (Figs. 1 and 2). The inhibitory rate of the high-dose Pu-erh tea extracts $(1.50 \mathrm{~g} / \mathrm{kg})$ was $71.52 \%$, which was higher than that of cimetidine $(46.73 \%)$. Meanwhile, gastric mucosal injury in rats pre-treated with green tea powder $(1.00 \mathrm{~g} / \mathrm{kg})$ was not significantly improved, though its inhibitory rate was $24.81 \%$.

Protective effect of the extracts against in gastric lesions based on histopathological evaluation. Histopathological assessment of the gastric tissues was subsequently performed (Fig. 3). Under high-power light microscopy, the gastric mucosa of rats in the normal control was smooth and flat. The layers of the gastric mucosa exhibited clear boundaries, and there were no signs of pathological changes such as hyperemia, bleeding or epithelial cell degeneration or necrosis (Fig. 3A). In the model control, however, relatively increased damage to the gastric mucosa was identified (Fig. 3B): The surface of the gastric mucosa was uneven and exhibited erosion, ulcers and bleeding; furthermore, marked pathological changes including hyperemia, bleeding and epithelial cell degeneration and necrosis were observed. Gastric mucosal damage in rats pre-treated with cimetidine or high-dose $\mathrm{Pu}$-erh tea extracts $(1.50 \mathrm{~g} / \mathrm{kg}$ ) was clearly alleviated (Fig. 3C and G), compared with that of the model control. Notably, the mucosal superficial layer of rats pre-treated with high-dose Pu-erh tea extracts was intact and the submucosal layers were only slightly congested (Fig. 3G). On scoring of the damage, the model group was determined to have a score for hyperemia of $1.22 \pm 0.16$, for bleeding of $2.11 \pm 0.37$, and for epithelial cell degeneration or necrosis of $3.33 \pm 0.25$ (Table III). Thus, the score for total pathological change in the model control was $15.44 \pm 0.56$, which was significantly higher than that of the normal control $(0.38 \pm 0.20 ; \mathrm{P}<0.05)$; whereas, the total scores for rats in the $\mathrm{Pu}$-erh tea extract groups were significantly decreased compared with the model group $(\mathrm{P}<0.05)$, to $9.75 \pm 1.16$ in the middle-dose group $(1.00 \mathrm{~g} / \mathrm{kg})$ and $6.22 \pm 0.77$ in the high-dose group $(1.50 \mathrm{~g} / \mathrm{kg}$; Table III). Green tea powder at the dose of $1.00 \mathrm{~g} / \mathrm{kg}$ did not exert significant protection in the gastric mucosa (Fig. 3D and Table III).

\section{Effect on MPO activity and ADMA concentration in mucosal} tissue. MPO activity in gastric tissue homogenate of the model control group was significantly increased compared with that of the normal control group $(2,032.59 \pm 69.63$ vs. $1,778.13 \pm 20.58 \mathrm{U} / 1, \mathrm{P}<0.05$; Fig. 4 ). The concentration of ADMA was also significantly higher in the model group compared with that in the normal control group $(1,411.25 \pm 20.85$ vs. $1,233.39 \pm 27.30 \mathrm{pmol} / \mathrm{ml}, \mathrm{P}<0.05$; Fig. 5$)$. The activity of MPO and the concentration of ADMA in rats pre-treated with cimetidine was $1,811.07 \pm 32.50 \mathrm{U} / 1$ (Fig. 4) and $1,242.37 \pm 24.76 \mathrm{pmol} / \mathrm{ml}$ (Fig. 5), respectively, which were reduced significantly compared with that of the model 
Table III. Histopathological scores of the gastric mucosa of rats with alcohol-induced gastric lesions pre-treated with the tested drugs.

\begin{tabular}{lcccc}
\hline Group & Hyperemia & Bleeding & Epithelial cell degeneration/necrosis & Pathological changes, total score \\
\hline Normal & $0.38 \pm 0.20$ & $0.00 \pm 0.00^{\mathrm{d}}$ & $0.00 \pm 0.00^{\mathrm{d}}$ & $0.38 \pm 0.20^{\mathrm{d}}$ \\
Model & $1.22 \pm 0.16^{\mathrm{a}}$ & $2.11 \pm 0.37^{\mathrm{b}}$ & $3.33 \pm 0.25^{\mathrm{b}}$ & $15.44 \pm 0.56^{\mathrm{b}}$ \\
Cimetidine $(\mathrm{g} / \mathrm{kg})$ & $1.11 \pm 0.12$ & $2.00 \pm 0.31$ & $1.56 \pm 0.31^{\mathrm{d}}$ & $9.78 \pm 0.79^{\mathrm{c}}$ \\
Green tea powder $(\mathrm{g} / \mathrm{kg})$ & $1.11 \pm 0.12$ & $1.67 \pm 0.35$ & $3.11 \pm 0.41$ & $13.78 \pm 1.50$ \\
Pu-erh tea extracts $(\mathrm{g} / \mathrm{kg})$ & & & & $13.89 \pm 1.26$ \\
0.5 & $1.11 \pm 0.12$ & $1.78 \pm 0.42$ & $3.44 \pm 0.26$ & $9.75 \pm 1.16^{\mathrm{c}}$ \\
1.0 & $1.25 \pm 0.25$ & $1.25 \pm 0.37$ & $2.00 \pm 0.76^{\mathrm{c}}$ & $6.22 \pm 0.77^{\mathrm{d}}$ \\
1.5 & $1.11 \pm 0.12$ & $0.56 \pm 0.19^{\mathrm{c}}$ & $1.33 \pm 0.18^{\mathrm{d}}$ & \\
\hline
\end{tabular}

Data were expressed as the mean \pm standard deviation. $(\mathrm{n}=9)$. ${ }^{\mathrm{a}} \mathrm{P}<0.05,{ }^{\mathrm{b}} \mathrm{P}<0.01$ vs. normal control group; ${ }^{\mathrm{c}} \mathrm{P}<0.05$, ${ }^{\mathrm{d}} \mathrm{P}<0.01 \mathrm{vs}$. model control group.

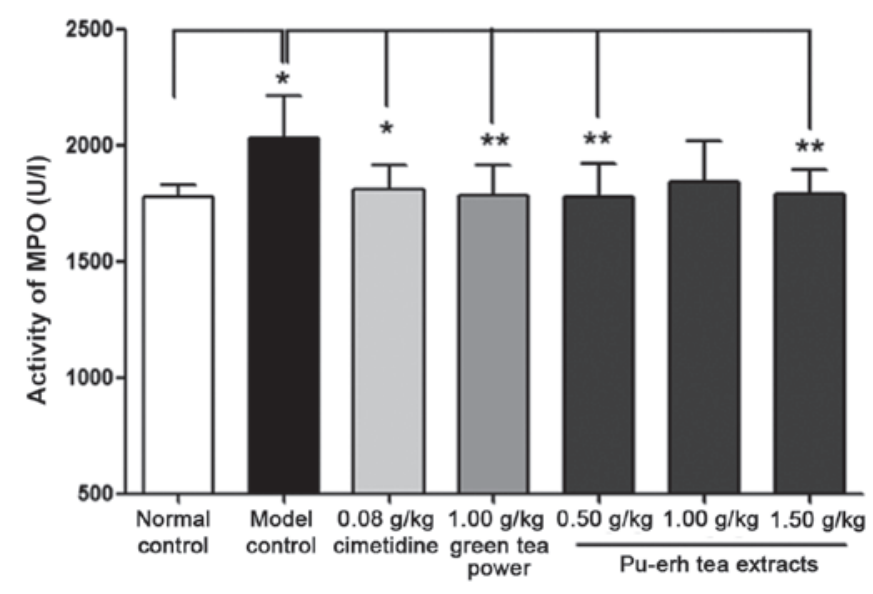

Figure 4. Effect of the tested drugs on MPO activity in the stomach tissues of rats with alcohol-induced gastric ulcer. Data were expressed as the mean \pm standard deviation $(n=9)$. ${ }^{*} \mathrm{P}<0.05$ and ${ }^{* *} \mathrm{P}<0.01$ as indicated. MPO, myeloperoxidase.

control group $(\mathrm{P}<0.05)$. Similarly, MPO activity in the low and high-dose $\mathrm{Pu}$-erh tea extract groups $(\mathrm{P}<0.01)$, and ADMA concentration in all three extract treatment groups $(0.50$ and $1.50 \mathrm{~g} / \mathrm{kg}$ : $\mathrm{P}<0.05 ; 1.00 \mathrm{~g} / \mathrm{kg}: \mathrm{P}<0.01)$ were also significantly attenuated compared with their levels in the model control group (Figs. 4 and 5).

\section{Discussion}

Gastric mucosal damage is caused by an imbalance between the protective and aggressive mechanisms in the mucosa, and is considered the net result of the actions of several endogenous factors and aggressive exogenous factors (11). The integrity of the gastric mucosa primarily depends on efficient protection of the gastric mucosal barrier, through maintaining defenses such as the gastric mucus layer, the mucus-bicarbonate barrier and mucosal microcirculation (14), which may be damaged by internal factors and external stimuli factors (11). Damage to the mucosa caused by internal factors and external stimuli is accompanied with the production of a number of inflamma-

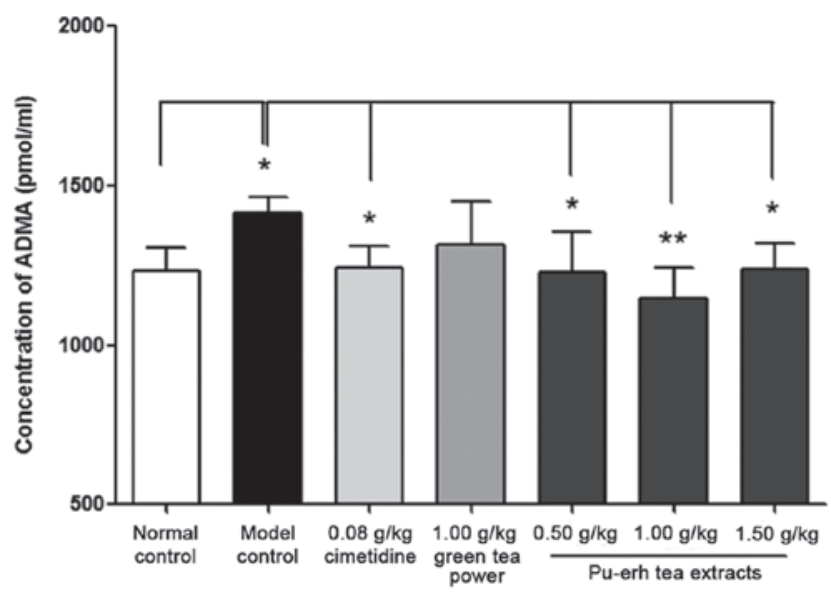

Figure 5. Effect of the tested drugs on ADMA concentration in the stomach tissues of rats with alcohol-induced gastric ulcer. Data were expressed as the mean \pm standard deviation $(n=9)$. ${ }^{*} \mathrm{P}<0.05$ and ${ }^{* *} \mathrm{P}<0.01$ as indicated. ADMA, asymmetric arginine.

tory mediators and cytokines (35). Gastric mucosal injury may occur when noxious factors attack the intact mucosal defense, or when the mucosal defensive mechanisms are impaired (14). The gastric mucus layer is the first line of defense that serves to protect stomach tissue from external stimuli (14). Mucosal defensive factors enable the mucosa to remain intact despite its frequent exposure to external substances and ranging temperature, $\mathrm{pH}$ and osmolarity, and notably, to substances with detergent or cytotoxic action and bacterial products that induce local and systemic inflammatory reactions $(36,37)$.

Ethanol is commonly used to induce ulcers in experimental animals, and causes acute gastric mucosal damage (38). Oral administration of absolute ethanol to rats produces typical characteristics of alcohol injury including linear hemorrhagic lesions, extensive submucosal edema, inflammatory cell infiltration and epithelial cell loss in stomach tissue (39). Ethanol produces necrotic lesions in the gastric mucosa through its direct toxic effect, as well as by reducing the secretion of bicarbonates and the production of mucus and defensive factors $(40,41)$. 
Gastric mucus when secreted in sufficient quantity is an important factor for the functioning of the gastric mucosa. It consists of a viscous, elastic, adherent and transparent gel, formed by water and glycoproteins, that covers the surface of the gastrointestinal mucosa (42). The protective properties of the mucus barrier depend not only on the gel structure but also on the amount or thickness of the layer covering the mucosal surface (28). In the present study, it was notable that folds of gastric mucosa were flattened and gastric damage was markedly reduced in rats pretreated with $\mathrm{Pu}$-erh tea extracts, compared with the model control. Furthermore, the pretreatment with $\mathrm{Pu}$-erh tea extracts at moderate and high-dose caused significant reduction in the UI and inhibition of gastric mucosal injury. A study by Scoparo et al (43) demonstrated that the fraction of green and black tea containing heteropolysaccharides reduced gastric lesions induced with ethanol and protected gastric mucosa tissue. In the current study, it was identified that $\mathrm{Pu}$-erh tea extracts clearly suppressed the formation of the gastric ulcer, and exhibited a higher inhibitory rate on gastric ulcer formation than green tea. This observed effect is probably associated with the high content of polysaccharides present in $\mathrm{Pu}$-erh tea extracts (43).

MPO, a biomarker for neutrophil-dependent inflammation, is mainly released from neutrophils, and therefore is also an essential marker for normal neutrophil function. MPO and other tissue-damaging substances including reactive oxygen metabolites and cytotoxic proteins are released into the extracellular space when neutrophils are stimulated $(44,45)$. Ethanol administration causes an increase of mucosal MPO activity, which thus indicates that the level of activated neutrophils secreting oxygen radicals is increased $(46,47)$. In the present study, absolute ethanol induced an increase of MPO activity in the model control compared with the normal control. The pretreatment of $\mathrm{Pu}$-erh tea extracts prior to this alcohol induction suppressed the release of MPO, compared with the model control, which may indicate that the degree of inflammation induced by neutrophils was also inhibited.

ADMA is the endogenous inhibitor of nitric oxide synthase (NOS), and has been implicated in pathophysiologies of the upper gastrointestinal tract (48). The generation of high levels of ADMA suppresses nitric oxide (NO) production through inhibition of NOS activity (49). NO, when serving as a potent vasodilator, increases blood flow in the gastric mucosa, inhibits the secretion of gastric acid and potentiates the secretion of mucus and bicarbonate, which thus protect the gastric mucosa against damage induced by a variety of corrosive substances and noxious agents (48). However, the biological life of ADMA is limited, as it may be hydrolyzed into L-citrulline and dimethylamine by dimethylarginine dimethylaminohydrolase (DDAH) excreted from the kidneys (50). A previous study focused on the effect of ADMA in gastric mucosal injury induced by ethanol, cold stress and indomethacin, and identified that ADMA levels were increased in gastric juice in animal models of gastric mucosal lesions (51). Furthermore, NO biosynthesis and DDAH activity in the stomach may be significantly inhibited in animals exposed to ethanol, stress and indomethacin (50-52). A study by Yi et al (53) study demonstrated that there were crude polyphenols in Dragon-pearl green tea, which increased the level of NO, improved microcirculation in the gastric mucosa, cleared oxygen free radicals and strengthened the protective function of the mucosal barrier. Adhikary et al (54) observed that both black tea and theaflavins suppressed various inflammatory modulators and inducible NOS-mediated nitric oxide synthesis during gastric ulcer healing. In the current study, the concentration of ADMA in the model control was significantly increased, compared with that in the normal control. The administration of $\mathrm{Pu}$-erh tea extracts prior to ethanol-induced injury exerted clear protective effects against the gastric mucosal injury, and the concentration of ADMA was significantly decreased, compared with the model control. Furthermore Pu-erh tea extracts were identified to decrease the concentration ADMA to a greater extent than green tea, which is probably due to the higher content of theaflavins in Pu-erh tea extracts (54).

In conclusion, the study present focused on the protective effect of Pu-erh tea extracts in the gastric mucosa. Absolute ethanol was used to induce gastric mucosal injury and the action of $\mathrm{Pu}$-erh tea extracts was investigated. The extracts exerted significant protective effects against the gastric mucosal damage. The human equivalent of the rat dose $1.00 \mathrm{~g} / \mathrm{kg}$ is $0.16 \mathrm{~g} / \mathrm{kg}$, according to the dose conversion relationship of the Food and Drug Administration (55), which is equivalent to physiological tea consumption in humans. The effect of Pu-erh tea extracts was greater than that of green tea powder. Pretreatment with $\mathrm{Pu}$-erh tea extracts prior to the intragastrical administration of absolute ethanol inhibited the activity of MPO and decreased the concentration of ADMA compared with the pre-administration of distilled water. These protective effects of $\mathrm{Pu}$-erh tea extracts against gastric mucosal damage may be due to its preservation of the gastric mucus layer as well as roles in decreasing inflammation and increasing NO production.

\section{Acknowledgements}

Not applicable.

\section{Funding}

The present study was supported by the China National Science and Technology Support Program (grant no. 2013Bad26q01).

\section{Availability of data and materials}

The analyzed data sets generated during the study are available from the corresponding author on reasonable request.

\section{Authors' contributions}

JY and WZ were the principal investigators responsible for the study and contributed to the design of the experiments, and JY wrote the original manuscript. YG and JD were responsible for analyzing the experimental data. XL, PT and YL were responsible for collecting experimental data. XM and $\mathrm{YZ}$ was the leader of the research group, and was responsible for the study design and guidance, and for checking the accuracy and authenticity of the manuscript. 


\section{Ethics approval and consent to participate}

The study protocols were approved by the Tasly Laboratory Animal Welfare and Ethics Committee of Tasly Pharmaceuticals, Inc. (Tianjin, China), and conducted according to the rules of animal experimentation and the guide for the Care and Use of Laboratory Animals of Tasly Pharmaceuticals, Inc.

\section{Consent for publication}

Not applicable.

\section{Competing interests}

WZ, XL, PT and YL are research fellows and XM is vice director of Tasly Pharmaceuticals, Inc., Tianjin, China. All other authors declare no competing interests.

\section{References}

1. Zhao M, Zhang DL, Su XQ, Duan SM, Wan JQ, Yuan WX, Liu BY, Ma Y and Pan YH: An Integrated Metagenomics/Metaproteomics Investigation of the Microbial Communities and Enzymes in Solid-state Fermentation of Pu-erh tea. Sci Rep 5: 10117, 2015.

2. Zhao L, Jia S, Tang W, Sheng J and Luo Y: Pu-erh tea inhibits tumor cell growth by down-regulating mutant p53. Int J Mol Sci 12: 7581-7593, 2011.

3. Syu KY, Lin CL, Huang HC and Lin JK: Determination of theanine, GABA, and other amino acids in green, oolong, black, and $\mathrm{Pu}$-erh teas with dabsylation and high-performance liquid chromatography. J Agric Food Chem 56: 7637-7643, 2008.

4. Fan JP, Fan C, Dong WM, Gao B, Yuan W and Gong JS: Free radical scavenging and anti-oxidative activities of an ethanol-soluble pigment extract prepared from fermented Zijuan Pu-erh tea. Food Chem Toxicol 59: 527-533, 2013.

5. Oi Y, Hou IC, Fujita $\mathrm{H}$ and Yazawa K: Antiobesity effects of Chinese black tea (Pu-erh tea) extract and gallic acid. Phytother Res 26: 475-481, 2012.

6. Zhang L, Shao WF, Yuan LF, Tu PF and Ma ZZ: Decreasing pro-inflammatory cytokine and reversing the immunosenescence with extracts of Pu-erh tea in senescence accelerated mouse (SAM). Food Chem 135: 2222-2228, 2012.

7. Hou Y, Shao W, Xiao R, Xu K, Ma Z, Johnstone BH and Du Y: $\mathrm{Pu}$-erh tea aqueous extracts lower atherosclerotic risk factors in a rat hyperlipidemia model. Exp Gerontol 44: 434-439, 2009.

8. Zhao X, Qian Y, Zhou YL, Wang R, Wang Q and Li GJ: Pu-erh tea has in vitro anticancer activity in TCA8113 cells and preventive effects on buccal mucosa cancer in U14 cells injected mice in vivo. Nutr Cancer 66: 1059-1069, 2014.

9. Pei S, Zhang Y, Xu H, Chen X and Chen S: Inhibition of the replication of hepatitis $\mathrm{B}$ virus in vitro by pu-erh tea extracts. J Agric Food Chem 59: 9927-9934, 2011.

10. Su Y, Zhang C, Wang Y and Li P: Antibacterial property and mechanism of a novel Pu-erh tea nanofibrous membrane. Appl Microbiol Biotechnol 93: 1663-1671, 2012.

11. Chen H, Liao H, Liu Y, Zheng Y, Wu X, Su Z, Zhang X, Lai Z, Lai X, Lin ZX and Su Z: Protective effects of pogostone from Pogostemonis Herba against ethanol-induced gastric ulcer in rats. Fitoterapia 100: 110-117, 2015.

12. Thomas D, Govindhan S,BaijuEC,Padmavathi G,Kunnumakkara $\mathrm{AB}$ and Padikkala J: Cyperus rotundus L. prevents non-steroidal anti-inflammatory drug-induced gastric mucosal damage by inhibiting oxidative stress. J Basic Clin Physiol Pharmacol 26: 485-490, 2015.

13. deFoneska A and Kaunitz JD: Gastroduodenal mucosal defense. Curr Opin Gastroenterol 26: 604-610, 2010.

14. Laine L, Takeuchi K and Tarnawski A: Gastric mucosal defense and cytoprotection: Bench to bedside. Gastroenterology 135: 41-60, 2008

15. Tarnawski AS, Ahluwalia A and Jones MK: The mechanisms of gastric mucosal injury: Focus on microvascular endothelium as a key target. Curr Med Chem 19: 4-15, 2012.
16. Brzozowski T,Ptak-Belowska A,Kwiecien S,Krzysiek-MaczkaG, Strzalka M, Drozdowicz D, Pajdo R, Olszanecki R, Korbut R, Konturek SJ, et al: Novel concept in the mechanism of injury and protection of gastric mucosa: Role of renin-angiotensin system and active metabolites of angiotensin. Curr Med Chem 19: 55-62, 2012.

17. Kuo KL, Weng MS, Chiang CT, Tsai YJ, Lin-Shiau SY and Lin JK: Comparative studies on the hypolipidemic and growth suppressive effects of oolong, black, pu-erh, and green tea leaves in rats. J Agric Food Chem 53: 480-489, 2005.

18. Antonisamy P, Kannan P, Aravinthan A, Duraipandiyan V, Arasu MV, Ignacimuthu S, Al-Dhabi NA and Kim JH: Gastroprotective activity of violacein isolated from Chromobacterium violaceum on indomethacin-induced gastric lesions in rats: Investigation of potential mechanisms of action. Sci World J 2014: 616432, 2014.

19. Li L, Luo XJ, Liu YZ, Zhang YS, Yuan Q, Tan N, Xiang DX and Peng J: The role of the DDAH-ADMA pathway in the protective effect of resveratrol analog BTM-0512 on gastric mucosal injury. Can J Physiol Pharmacol 88: 562-567, 2010.

20. Hou CW, Jeng KC and Chen YS: Enhancement of fermentation process in Pu-erh tea by tea-leaf extract. J Food Sci 75: H44-H48, 2010.

21. Jeng KC, Chen CS, Fang YP, Hou RC and Chen YS: Effect of microbial fermentation on content of statin, GABA, and polyphenols in Pu-Erh tea. J Agric Food Chem 55: 8787-8792, 2007.

22. Duh PD, Yen GC, Yen WJ, Wang BS and Chang LW: Effects of pu-erh tea on oxidative damage and nitric oxide scavenging. J Agric Food Chem 52: 8169-8176, 2004.

23. Xu Y, Zhao H, Zhang M, Li CJ, Lin XZ, Sheng J and Shi W: Variations of antioxidant properties and NO scavenging abilities during fermentation of tea. Int J Mol Sci 12: 4574-4590, 2011.

24. Chen YS, Liu BL and Chang YN: Bioactivities and sensory evaluation of $\mathrm{Pu}$-erh teas made from three tea leaves in an improved pile fermentation process. J Biosci Bioeng 109: 557-563, 2010.

25. Wang Q, Gong J, Chisti Y and Sirisansaneeyakul S: Fungal isolates from a Pu-erh type tea fermentation and their ability to convert tea polyphenols to theabrownins. J Food Sci 80: M809-M817, 2015.

26. Xu Y, Wang G, Li C, Zhang M, Zhao H, Sheng J and Shi W: $\mathrm{Pu}$-erh tea reduces nitric oxide levels in rats by inhibiting inducible nitric oxide synthase expression through toll-like receptor 4. Int J Mol Sci 13: 7174-7185, 2012.

27. Liu CF, Lin CC, Ng LT and Lin SC: Protection by tetramethylpyrazine in acute absolute ethanol-induced gastric lesions. J Biomed Sci 9: 395-400, 2002.

28. Hua HE: Application of Anaesthesia with Combined Ether Inhalation and Sodium Pentobarbital Intraperitoneal Injection to Rat Liver Transplantation. Chin J Comp Med 18: 30-31, 2008 (In Chinese).

29. Halim SZ, Zakaria ZA, Omar MH, Mohtarrudin N, Wahab IRA and Abdullah MNH: Synergistic gastroprotective activity of methanolic extract of a mixture of Melastoma malabathricum and Muntingia calabura leaves in rats. BMC Complement Altern Med 17: 488, 2017.

30. Balan T, Sani MH, Mumtaz Ahmad SH, Suppaiah V, Mohtarrudin N and Zakaria ZA: Antioxidant and anti-inflammatory activities contribute to the prophylactic effect of semi-purified fractions obtained from the crude methanol extract of Muntingia calabura leaves against gastric ulceration in rats. J Ethnopharmacol 164: 1-15, 2015.

31. Balan T, Mohd Sani MH, Suppaiah V, Mohtarrudin N, Suhaili Z, Ahmad Z and Zakaria ZA: Antiulcer activity of Muntingia calabura leaves involves the modulation of endogenous nitric oxide and nonprotein sulfhydryl compounds. Pharm Biol, 2013.

32. Technical Standards for Testing and Assessment of Health Food: Document Guidelines of the Ministry of health of the People's Republic of China, pp23-28, 2012.

33. Guth PH, Aures D and Paulsen G: Topical aspirin plus $\mathrm{HCl}$ gastric lesions in the rat. Cytoprotective effect of prostaglandin, cimetidine, and probanthine. Gastroenterology 76: 88-93, 1979.

34. Li Q, Yang LL, Fan LL, Liang C, Wang QJ, Wen HM, Dai JW, Li X and Zhang YY: Activity of Brucea javanica oil emulsion against gastric ulcers in rodents. Asian J Pharmaceutical Sci (In press).

35. Mei X, Xu D, Xu S, Zheng Y and Xu S: Novel role of $\mathrm{Zn}(\mathrm{II})$-curcumin in enhancing cell proliferation and adjusting proinflammatory cytokine-mediated oxidative damage of ethanol-induced acute gastric ulcers. Chem Biol Interact 197: 31-39, 2012. 
36. Alrashdi AS, Salama SM, Alkiyumi SS, Abdulla MA, Hadi AH, Abdelwahab SI, Taha MM, Hussiani J and Asykin N: Mechanisms of gastroprotective effects of ethanolic leaf extract of Jasminum sambac against $\mathrm{HCl} /$ ethanol-induced gastric mucosal injury in rats. Evid Based Complement Alternat Med 2012: 786426, 2012.

37. Hashim H, Mughrabi FF, Ameen M, Khaledi $\mathrm{H}$ and Ali HM: Cytoprotective effect of benzyl N'-(5-chloro-indol-3-yl-methylidene)-hydrazinecarbodithioate against ethanol-induced gastric mucosal injury in rats. Molecules 17: 9306-9320, 2012.

38. Park CH, Nam DY, Son HU, Lee SR, Lee HJ, Heo JC, Cha TY, Baek JH and Lee SH: Polymer fraction of Aloe vera exhibits a protective activity on ethanol-induced gastric lesions. Int J Mol Med 27: 511-518, 2011.

39. Gazzieri D, Trevisani M, Springer J, Harrison S, Cottrell GS Andre E, Nicoletti P, Massi D, Zecchi S and Nosi D: Substance P released by TRPV1-expressing neurons produces reactive oxygen species that mediate ethanol-induced gastric injury. Free Radic Biol Med 43: 581-589, 2007.

40. Luiz-Ferreira A, Almeida AC, Cola M, Barbastefano V, Almeida AB, Batista LM, Farias-Silva E, Pellizzon $\mathrm{CH}$ Hiruma-Lima CA, Santos LC, et al: Mechanisms of the gastric antiulcerogenic activity of Anacardium humile St. Hil on ethanol-induced acute gastric mucosal injury in rats. Molecules 15: 7153-7166, 2010.

41. Ibrahim IA, Qader SW, Abdulla MA, Nimir AR, Abdelwahab SI and Al-Bayaty FH: Effects of Pithecellobium jiringa ethanol extract against ethanol-induced gastric mucosal injuries in Sprague-Dawley rats. Molecules 17: 2796-2811, 2012.

42. Tulassay $\mathrm{Z}$ and Herszényi L: Gastric mucosal defense and cytoprotection. Best Pract Res Clin Gastroenterol 24: 99-108, 2010.

43. Scoparo CT, Souza LM, Dartora N, Sassaki GL, Santana-Filho AP, Werner MF, Borato DG, Baggio CH and Iacomini M: Chemical characterization of heteropolysaccharides from green and black teas (Camellia sinensis) and their anti-ulcer effect. Int J Biol Macromol 86: 772-781, 2016.

44. Nartey ET, Ofosuhene M and Agbale CM: Anti-ulcerogenic activity of the root bark extract of the African laburnum 'Cassia sieberiana' and its effect on the anti-oxidant defence system in rats. BMC Complement Altern Med 12: 247, 2012.

45. Zhao W, Zhu F, Shen W, Fu A, Zheng L, Yan Z, Zhao L and Fu G: Protective effects of DIDS against ethanol-induced gastric mucosal injury in rats. Acta Biochim Biophys Sin (Shanghai) 41: 301-308, 2009

46. Devi RS, Narayan S, Vani G and Shyamala Devi CS Gastroprotective effect of Terminalia arjuna bark on diclofenac sodium induced gastric ulcer. Chem Biol Interact 167: 71-83, 2007.
47. Santos Cerqueira G, dos Santos e Silva G, Rios Vasconcelos E, Fragoso de Freitas AP, Arcanjo Moura B, Silveira Macedo D, Lopes Souto A, Barbosa Filho JM, de Almeida Leal LK, de Castro Brito GA, et al: Effects of hecogenin and its possible mechanism of action on experimental models of gastric ulcer in mice. Eur J Pharmacol 683: 260-269, 2012.

48. Szlachcic A, Krzysiek-Maczka G, Pajdo R, Targosz A, Magierowski M, Jasnos K, Drozdowicz D, Kwiecien S and Brzozowski T: The impact of asymmetric dimethylarginine (ADAMA), the endogenous nitric oxide (NO) synthase inhibitor, to the pathogenesis of gastric mucosal damage. Curr Pharm Des 19: 90-97, 2013.

49. Tran CT, Leiper JM and Vallance P: The DDAH/ADMA/NOS pathway. Atheroscler Suppl 4: 33-40, 2003.

50. Zhang Z, Zou YY, Li FJ and Hu CP: Asymmetric dimethylarginine: A novel biomarker of gastric mucosal injury? World $\mathrm{J}$ Gastroenterol 17: 2178-2180, 2011

51. Zhang Z, Zhou Y, Zou YY, Wang L, Yang ZC, Guo R, Li D, Peng J and Li YJ: Detrimental effects of nicotine on the acute gastric mucosal injury induced by ethanol: Role of asymmetric dimethylarginine. Can J Physiol Pharmacol 86: 835-840, 2008.

52. Kwiecien S, Ptak-Belowska A, Krzysiek-Maczka G, Targosz A, Jasnos K, Magierowski M, Szczyrk U, Brzozowski B, Konturek SJ, Konturek PC, et al: Asymmetric dimethylarginine, an endogenous inhibitor of nitric oxide synthase, interacts with gastric oxidative metabolism and enhances stress-induced gastric lesions. J Physiol Pharmacol 63: 515-524, 2012.

53. Yi R, Wang R, Sun P and Zhao X: Antioxidant-mediated preventative effect of Dragon-pearl tea crude polyphenol extract on reserpine-induced gastric ulcers. Exp Ther Med 10: 338-344, 2015.

54. Adhikary B, Yadav SK, Chand S, Bandyopadhyay SK and Chattopadhyay S: Black tea and theaflavins suppress various inflammatory modulators and i-NOS mediated nitric oxide synthesis during gastric ulcer healing. Free Radic Res 45: 767-778, 2011.

55. Food and Drug Administration (FDA): Estimating the safe starting dose in clinical trials for therapeutics in adult healthy volunteers. Guidance for Industry and Reviewers. FDA, Rockville, MD, p26, 2002.
This work is licensed under a Creative Commons Attribution-NonCommercial-NoDerivatives 4.0 International (CC BY-NC-ND 4.0) License. 